\title{
Controversies in Mucosal Healing in Ulcerative Colitis
}

\author{
Sunanda Kane, MD, ${ }^{*}$ Frances $L u, M D,{ }^{+}$Asher Kornbluth, $M D,{ }^{+}$Dahlia Awais, $M D,{ }^{\neq}$and \\ Peter D.R. Higgins, MD, PhD, MSC
}

\section{ENDOSCOPIC HEALING SHOULD BE A GOAL FOR EVERYONE WITH ULCERATIVE COLITIS: PRO}

\author{
Sunanda Kane, MS
}

A clinicians we would all agree that the goal for our patients is to induce remission, which is usually defined as symptom resolution. Restoring continence and absence of rectal bleeding would be signs that the mucosa is at most minimally inflamed if not healed. However, there have not been any data to confirm that symptomatic improvement alters the natural history of the disease, nor decreases the lifetime risk for surgery. Because we understand the potential complications of this disease, it should not be enough that we "settle" for symptom resolution, but aim instead to decrease the risk of complications and alter the natural history of the disease.

Until recently, mucosal healing endpoints have not been included in clinical trials. Clinical endpoints lead to high placebo responses, potentially diluting the effect of the therapy, even in large multinational trials. The hard endpoint of endoscopic mucosal healing reduces the number needed to power a study for treatment effect.

The relevant questions during this discussion are: 1) What is endoscopic healing? 2) How often can we achieve this in ulcerative colitis (UC)? 3) Does mucosal healing change disease outcomes? and 4) Does mucosal healing lower the rate of complications?

The history of mucosal healing starts in 2001 when Bitton et $\mathrm{al}^{1}$ reported on clinical, biological, and histologic parameters that would predict time to clinical relapse. One of the only factors that was statistically significant was basal plasmacytosis on rectal biopsy. The hazard ratio (HR) for

Received for publication September 25, 2008; Accepted December 15, 2008.

From the *Mayo Clinic, Rochester, Minnesota, ${ }^{\dagger}$ Mount Sinai Hospital, New York, New York, " University of Michigan Hospital, Ann Arbor, Michigan.

Reprints: Dr. Peter Higgins, Box 0682, 1150 West Medical Center Dr., Ann Arbor, MI 48109 (e-mail: phiggins@umich.edu).

Copyright (C) 2009 Crohn's \& Colitis Foundation of America, Inc.

DOI 10.1002/ibd.20875

Published online 11 February 2009 in Wiley InterScience (www. interscience.wiley.com). predicting clinical relapse was 4.5 and the authors concluded that this factor may help identify patients with inactive UC who will require optimal maintenance therapy.

An accepted definition of endoscopic healing has yet to be developed and validated. Everyone would agree that return to normal vascular pattern, the absence of friability, or ulcerations fits the bill, but what about some mild erythema or granularity? If there is no friability (however you want to measure it), should that suffice? Mucosal healing was defined as either completely normal (score of 0 ) or mild (score of 1 ) in the ASCEND and ACT trials but $<1$ in the MMX (mesalamine) trials.

Using the ASCEND criteria, mucosal healing was achieved in up to $80 \%$ of those patients on $4.8 \mathrm{~g} /$ mesalamine/day at week $6 .{ }^{2,3}$ Using the stricter subscore of 0 , then this percentage fell to $\approx 30 \%$. In the MMX trials, $77.6 \%$ of patients had a score of $<1$ on $4.8 \mathrm{~g} /$ day at the end of the trial at 12 weeks. ${ }^{4,5}$ In the infliximab trial, healing was achieved by $62 \%$ of the $5 \mathrm{mg} / \mathrm{kg}$ dose at week 8 , and was sustained in $45.5 \%$ of patients at week $54 .^{6}$ If a score of 0 or 1 is acceptable, then certainly mucosal healing as an endpoint is an achievable and reasonable goal.

Just because the majority of patients may achieve mucosal healing, does it make any difference? In the ACT trial, those patients with documented mucosal healing at week 8 and 30 were more likely to be in remission than those who did not $(P=0.009) .{ }^{6}$ We do not have such data from the mesalamine trials, as endoscopy at the end of the trial was not part of the protocol. It would, however, make intuitive sense that those patients without any symptoms at the end of the trial would most likely have scores of 1 or 0 . In a currently ongoing prospective study, preliminary results from patients with active UC were presented. ${ }^{7}$ Patients with mild to moderate UC were treated with 6 weeks of $4 \mathrm{~g} /$ day oral and $2 \mathrm{~g}$ enemas. In 78 patients, 59 achieved clinical remission. Endoscopic activity was still present in only 5 . After a mean follow-up of 8.7 months, 15 (26\%) relapsed. The cumulative rate of relapse at 1 year was $23 \%$ in patients with clinical and endoscopic remission and $80 \%$ in patients with only clinical remission. The conclusion of the authors was that persistence of endoscopic activity is a rare event, with active UC achieving clinical remission after 6 weeks, but a very strong predictor of early relapse.

Data from the IBSEN study in Norway followed incident cases of UC over a period of 5 years. ${ }^{8}$ In all, 354 patients were endoscoped at 6 months to 2 years after their diagnosis, 328 
reevaluated 5 years after diagnosis, and 150 greater than 5 years after diagnosis. At 1 year, 3 patients with mucosal healing documented underwent surgery versus the 13 in those with mucosal healing. Those with healing had a $78 \%$ less chance of undergoing colectomy than those with active disease on endoscopy after 1 year (odds ratio [OR] $0.22,95 \%$ confidence interval [CI] 0.06-0.79). Other clinical outcomes included a protective effect of mucosal healing on severe complaints at 5 years with an OR of 0.39 (95\% CI 0.16-0.94).

If we take the argument a step further and say that endoscopic healing is not enough, but a marker for histologic healing, then we can talk about information regarding other outcomes. There are 2 retrospective studies to support the belief that inflammation is a risk factor for colorectal cancer (CRC). ${ }^{9,10}$ The St. Mark's surveillance database has demonstrated that histologic inflammation was associated with a 5 times greater likelihood of CRC, endoscopic inflammation with 2.5 , and on multivariate analysis histologic inflammation with OR 4.7. ${ }^{9}$ In another study from Mt Sinai, 15 out of 418 patients in a surveillance program progressed to advanced neoplasia. Sixty-five of 418 progressed to any neoplasia. The mean histologic inflammation over time and progression to advanced neoplasia was 3.0 (95\% CI 1.4-6.3). ${ }^{10}$

There has also been recent work by Rubin et $\mathrm{al}^{11}$ at the University of Chicago. Using a new histologic grading scale, mean inflammation score was positively correlated with an increased risk of cancer (OR 2.6, 95\% CI 1.5-4.5). Taking into consideration the presence of pancolitis, the OR was $2.3(95 \%$ CI 1.3-4.2). There was also a dose response with unit increases of inflammation incurring a higher risk. There was also an increased risk for colectomy with an HR of 1.9 (95\% CI 1.03.5). ${ }^{12}$ The proposed biologic mechanism for these findings includes evidence for inflammation through increased proinflammatory cytokines of IL-2313 and NF-kappa B. ${ }^{14}$

Since it is possible to achieve endoscopic healing in the majority of patients, and it appears that this may be related to improved long-term outcomes, our new goals for management of UC should be to provide validated indices to measure mucosal healing, and determine the optimal time point to assess mucosal healing.

\section{MUCOSAL HEALING IS NOT AN IMPORTANT ENDPOINT FOR PATIENTS WITH ULCERATIVE COLITIS: CON}

\section{Frances $L u, M D$, and Asher Kornbluth, MD}

There are currently no universally accepted or validated endpoints for measuring the efficacy of therapy for UC. Typical primary and secondary endpoints include clinical improvement, clinical remission, and most recently, mucosal healing as defined endoscopically. However, the importance of mucosal healing as a primary endpoint, and as a prognosticator of likelihood of recurrence, remains unknown. There are a number of questions
TABLE 1. The Mayo Clinic Endoscopic Score

$$
\begin{aligned}
0= & \text { Normal or inactive disease } \\
1= & \text { Mild disease: erythema, decreased vascular pattern, mild } \\
& \text { friability } \\
2= & \text { Moderate disease: marked erythema, absent vascular pattern, } \\
& \text { friability, erosions } \\
3= & \text { Severe disease: spontaneous bleeding, ulceration }
\end{aligned}
$$

to address before granting mucosal healing primacy in assessing disease activity. These include: What is the definition of mucosal healing - is there a uniformly accepted standard? Is mucosal healing necessary to monitor disease activity? Does it predict the future disease course? Does mucosal healing reduce the likelihood of future dysplasia and CRC?

\section{What Is the Definition of Mucosal Healing?}

Different instruments have been developed over the past 50 years to assess disease activity in UC based on endoscopic evaluation of mucosal appearance. Each of these instruments is described in depth by D'Haens et $\mathrm{al}^{15}$ in an extensive review of the indices used in clinical trials of medical therapy in UC. Despite 50 years of clinical trials and a total of 14 (!) differently designed endoscopic scoring systems, the definitions and the scoring methods of these instruments have never been validated. These scoring systems employ scales that range from 3-point systems ${ }^{16}$ to 12-point systems..$^{17}$ Scores have been based on endoscopic appearance ranging from a rigid sigmoidoscopic view to more extensive video imaging. Interobserver variability kappa scores of these scales have never been compared between scoring systems. Furthermore, there is no agreement as to what absolute score or degree of change from baseline score constitutes disease response or remission.

For example, the recent pivotal trials of infliximab for moderate-severe UC, ${ }^{6}$ utilized the Mayo score ${ }^{18}$ (Table 1), for which the endoscopic subscore allows a score of 0 or 1 to be considered as mucosal healing; this therefore allows patients with continued mucosal edema, loss of vascularity, and friability to be granted the claim of mucosal healing. Based on this disingenuous definition, in infliximab-treated patients so-called mucosal healing occurred $25 \%$ more frequently than patients actually achieving clinical remission, $62 \%$ versus $37 \%$, respectively, at week 8 , after 3 infusions.

In 2007 the AGA published its "Consensus on Efficacy End Points," stating that "absence of friability, blood, erosions, and ulcers in all visualized segments are the required components of genuine endoscopic healing." (italics added). ${ }^{15}$ Similarly, regarding microscopic mucosal healing, "the authors do not recommend that histologic remission be used as the primary end point for a therapeutic trial in patients with UC." 


\section{Is Mucosal Healing Necessary to Monitor Disease Activity?}

In clinical practice, patient awareness of their usual typical flare symptoms usually obviates the need for endoscopic confirmation. This was studied by Higgins et al ${ }^{19}$ in 66 consecutive patients who were evaluated with 2 indices utilizing sigmoidoscopic scores ${ }^{18,20}$ and compared it to 2 noninvasive indices not utilizing sigmoidoscopic scores..$^{21,22}$ Linear regression analysis demonstrated a very strong correlation between the clinical symptom index and the endoscopic score $(r=0.7980)$. Endoscopic items contributed very little $(0.04 \%-3.37 \%)$ to the variability in the measurement of disease activity. These findings support the clinical practice of treating patients based on symptom reporting without endoscopy. Manes et al, ${ }^{23}$ in a prospective series of 507 patients with UC undergoing colonoscopy, similarly found that the endoscopic findings influenced management in only $7.6 \%$ of cases. A strong correlation between severity of symptoms and degree of mucosal lesions was demonstrated, consistent with the conclusion that the finding of mucosal lesions can be predicted based on the patients' clinical symptoms.

In a meta-analysis of all placebo-controlled trials in UC published in 2007, 6 trials were found that included endoscopic remission rates as an endpoint in combination with clinical remission. In 4 of 6 of these trials the endoscopic remission rate was identical to the clinical remission rate. This finding further supports the viewpoint that the endoscopic assessment of mucosal healing did not yield additional clinical information beyond that supplied by the patient. ${ }^{24}$

\section{Does Mucosal Healing Predict the Future Clinical Course?}

A study that is certain to be cited as evidence that mucosal healing as an important predictor of future course is the series reported by Froslie et al. ${ }^{8}$ This study analyzed a Norwegian cohort of 495 patients from the years of 19901994 and followed these patients for 5 years. However, a critical analysis of the design of this series makes it impossible to support their conclusion that mucosal healing is predictive of a milder course of disease.

The study design assessed mucosal healing on a single day 1 year after the patient was enrolled in the study with active disease. The patient may have been on any therapy, for any duration, during any interval before that single sigmoidoscopy on that single day in time. Furthermore, there was no control for interobserver variability between the endoscopists.

At the 5-year visit, all participants were asked to categorize their disease course into 1 of 4 predefined values: 1) remission after initial activity, 2) continuous activity, 3) relapsing disease, and 4) accelerating disease activity. The study did find a lower colectomy rate in patients with milder mucosal inflammation on that single sigmoidoscopy 4 years earlier. However, there was no reduction in: number of re- lapses, overall 5-year disease activity, use of oral steroids at any time, and no reduction in likelihood of disease extension. The conclusion that mucosal healing (on a single day) is predictive of future clinical course cannot at all be supported based on this large cohort of patients followed prospectively.

\section{Does Mucosal Healing Reduce the Likelihood of Future Dysplasia and CRC?}

There are a number of recent series suggesting that increased mucosal inflammation increases the risk of subsequently developing dysplasia and CRC. In a series of prospectively collected surveillance data, Rutter et $\mathrm{al}^{25}$ found that macroscopically visible features of severe acute and chronic inflammation, as well as features indicative of a previous state of severe inflammation, were associated with a significant increase in the risk for colorectal neoplasia. However, when these data were analyzed in a multivariate analysis, macroscopic inflammation as described by the endoscopist was no longer significantly associated with future dysplasia or CRC, and it was only microscopic inflammation that was associated with a greater subsequent risk of dysplasia and CRC.

Similarly, 2 additional large series from the Mt Sinai ${ }^{10}$ and the University of $\mathrm{Chicago}^{11}$ groups found a reduced risk of dysplasia and CRC in patients with lesser histologic inflammation. No data, however, was presented regarding endoscopic mucosal healing at any timepoint. However, as discussed above there is no validated histologic score among the 14 different histology scores utilized in clinical series ${ }^{15}$ and the studies of Rutter et al, Gupta et al, and Rubin et al in fact introduced 3 additional histologic scoring systems.

While it may be intellectually intuitive and visually tempting to include an endoscopic assessment in evaluating patients with active UC, mucosal healing has so far not been: uniformly defined, found to yield additional information to that provided by a patient history, proven to predict future disease course, or reduce dysplasia or CRC risk based on endoscopic appearance alone. At present, therefore, it has not yet demonstrated its importance as an endpoint for assessing patients with UC.

\section{MUCOSAL HEALING IS GOOD IN UC BUT IT IS NOT YET CLEAR HOW TO BEST MEASURE IT, HOW MUCH HEALING IS IMPORTANT, AND WHETHER CHANGING THERAPY FOR A FEW ULCERS MATTERS: BALANCE}

\section{Dahlia Awais, MD, and \\ Peter D.R. Higgins, MD, PhD, MSC}

The significance of mucosal healing as a treatment goal in IBD is of increasing interest and has become a common endpoint in clinical trials; however, its actual importance as an endpoint with value beyond careful symptom evaluation has not yet been proven. The goal of any therapeutic intervention is to decrease morbidity, mortality, or both. In IBD 
we eagerly await the discovery of that feature, biomarker, or gene which will allow us to predict an individual patient's future course and their risk for steroids, hospitalizations, dysplasia, or surgery. Ideally this predictor would be cheap and noninvasive; nonetheless, in the absence of better alternatives (more accurate, cheaper, less invasive), if mucosal healing is predictive of a decreased risk of any of these its importance will be self-evident. At this point, although the idea of mucosal healing as such a predictor has intuitive appeal (we are after all talking about a mucosal disease), the data, in fact, remain limited. More data from rigorously conducted studies are needed before any definitive statements can be made on the value of mucosal healing in UC.

In this issue of Inflammatory Bowel Diseases Drs. $\mathrm{Kane}, \mathrm{Lu}$, and Kornbluth eloquently argue for and against mucosal healing as a treatment goal in UC in 2009. Dr. Kane argues that endoscopic healing is an achievable goal. She argues that its absence is a strong predictor of relapse and that it may be associated with better outcomes. Drs. Lu and Kornbluth take the opposite side and argue the data supporting improved outcomes with endoscopic healing is actually quite weak, and that endoscopic findings do not provide more information than that which can be understood from patient clinical symptoms alone. Both sides, however, agree that a validated measure of mucosal healing has not been established but is necessary for an optimal understanding of the significance of mucosal healing as an endpoint. And both sides would agree that the available data on the significance of mucosal healing are limited.

In clinical practice, treatment response is based on symptoms, with endoscopy reserved for the patients who do not seem to be responding. No one would dispute the importance of symptom resolution as a therapeutic goal, as symptoms have a direct impact on patient quality of life. Mucosal healing is less tangible to the patient. Furthermore, endoscopy is invasive, costly, and can be distressing to patients. Therefore, high-quality evidence that it predicts long-term important outcomes such as flare, steroid use, surgery, or dysplasia are critical in assessing the importance of mucosal healing as an endpoint. So, do we have high-quality evidence?

The impact of mucosal healing was studied by Froslie et $\mathrm{al}^{8}$ in a Norwegian cohort over 5 years. They concluded that mucosal healing is predictive of disease course. They report lower rates of colectomy at 5 years in patients with documented mucosal healing compared to those with active disease on endoscopy; however, they found no significant relationship between mucosal healing and other outcomes such as future disease activity, steroid use, or future mucosal healing. Although the findings are provocative, our interpretation and understanding must be informed by the limitations of this study. The conclusion that mucosal healing predicts disease course is in fact not supported by the results, most of which revealed a lack of association with future outcomes, except for that of colectomy. Furthermore, given the number of associations explored, there is a high risk for type I errors, so that even the association between mucosal healing and colectomy may have been a mere chance association, bringing the validity of the findings into question. In addition, neither patients nor physicians were blinded to the endoscopy findings, and these may have influenced colectomy decisions, producing a bias in favor of the importance of mucosal healing. A final criticism of this article is that mucosal healing was not compared to reasonable, less invasive surrogate markers as a predictor of outcomes. Clinical symptoms, stool blood, or fecal markers of inflammation may have been just as good or better prognostic markers without the cost or invasive nature of colonoscopy.

We have more convincing data to support an association between inflammation and the development of dysplasia and colorectal cancer. A case-control study by Rutter et $\mathrm{al}^{9}$ found that endoscopic features of previous inflammation such as inflammatory polyps were associated with an increased risk of neoplasia, while normal mucosa on endoscopy was highly correlated with a decreased risk. We do have additional information supporting a relationship between histological inflammation and neoplasia ${ }^{10,25}$; however, while macroscopic inflammation may be a marker for microscopic inflammation, they are not one and the same.

In 2009, our best data suggests that endoscopic mucosal healing likely decreases the risk for neoplasia, but may or may not be adequate if histologic healing is what is really required. Regarding other outcomes such as future flare, steroid use, or surgery, current data are too limited. More studies are needed to answer these questions. And in order to answer these questions well, a validated measure of endoscopic disease activity (something we still do not have) is required. Such a scoring system would need to be reproducible, responsive to change, and have low interobserver variability.

Questions regarding mucosal healing can be answered with the right study. We would like to see a prospective study in which a validated measure of endoscopic disease activity is used to evaluate endoscopy videos of patients in clinical remission. Reviewers would be blinded to patient information, and patients and their physicians would be blinded to the endoscopic score. Inexpensive, noninvasive prognostic alternative markers would also be collected, including careful evaluation of patient symptoms, signs (including stool blood), and fecal and blood biomarkers. Outcomes could then be assessed 12 months later. Controlling for covariates, the importance of mucosal healing in future outcomes could be assessed compared to noninvasive predictors. If mucosal healing is important, and provides additional prognostic information, additional studies would be necessary to determine what level of mucosal injury would justify a change in therapy in a minimally symptomatic patient. 
Mucosal healing may prove to be an important surrogate endpoint with great prognostic value. Our currently available data are inadequate to prove this. Future studies using a validated scoring system would be helpful in better understanding possible associations between mucosal healing and important outcomes. Mucosal healing, or any other therapeutic goal, must always include consideration of the importance of the benefit versus the additional costs and potential risks incurred when changing therapy. The true importance of mucosal healing as a surrogate endpoint in clinical trials, and whether incomplete mucosal healing justifies a change in therapy, requires further study.

\section{REFERENCES}

1. Bitton A, Peppercorn MA, Antonioli DA, et al. Clinical, biological, and histologic parameters as predictors of relapse in ulcerative colitis. Gastroenterology. 2001;120:13-20.

2. Hanauer SB, Sandborn WJ, Kornbluth A, et al. Delayed-release oral mesalamine at $4.8 \mathrm{~g} /$ day ( $800 \mathrm{mg}$ tablet) for the treatment of moderately active ulcerative colitis: the ASCEND II trial. Am J Gastroenterol. 2005; 100:2478-2485.

3. Hanauer SB, Sandborn WJ, Dallaire C, et al. Delayed-release oral mesalamine $4.8 \mathrm{~g} / \mathrm{day}$ ( $800 \mathrm{mg}$ tablets) compared to $2.4 \mathrm{~g} /$ day (400 mg tablets) for the treatment of mildly to moderately active ulcerative colitis: The ASCEND I trial. Can J Gastroenterol. 2007;21:827-834.

4. Kamm MA, Sandborn WJ, Gassull M, et al. Once-daily, high-concentration MMX mesalamine in active ulcerative colitis. Gastroenterology. 2007;132:66-75; quiz 432-433.

5. Lichtenstein GR, Kamm MA, Boddu P, et al. Effect of once- or twicedaily MMX mesalamine (SPD476) for the induction of remission of mild to moderately active ulcerative colitis. Clin Gastroenterol Hepatol. 2007;5:95-102

6. Rutgeerts P, Sandborn WJ, Feagan BG, et al. Infliximab for induction and maintenance therapy for ulcerative colitis [see Comment]. $N$ Engl J Med. 2005;353:2462-2476 [erratum, N Engl J Med 2006;354:2200].

7. Meucci G, Fasoli R, Saibeni S, et al. Prognostic significance of endoscopic remission in patients with active ulcerative colitis treated with oral and topical mesalazine: preliminary results from a prospective, multicenter study. In: Association AG, ed. Digestive Disease Week. Chicago; 2006:A197.

8. Froslie KF, Jahnsen J, Moum BA, et al. Mucosal healing in inflammatory bowel disease: results from a Norwegian population-based cohort. Gastroenterology. 2007;133:412-422.

9. Rutter MD, Saunders BP, Wilkinson $\mathrm{KH}$, et al. Cancer surveillance in longstanding ulcerative colitis: endoscopic appearances help predict cancer risk. Gut. 2004;53:1813-1816.

10. Gupta RB, Harpaz N, Itzkowitz S, et al. Histologic inflammation is a risk factor for progression to colorectal neoplasia in ulcerative colitis: a cohort study. Gastroenterology. 2007;133:1099-1105.

11. Rubin D, Huo D, Rothe J, et al. Increased inflammatory activity is an independent risk factor for dysplasia and colorectal cancer in ulcerative colitis: a case-control analysis with blinded prospective pathology review. Digestive Disease Week. Gastroenterology; 2005:A103.

12. Rubin D, Huo D, Hetzel $J$, et al. Increased degree of histological inflammation predicts colectomy and hospitalization in patients with ulcerative colitis. Gastroenterology: Digestive Disease Week 2007:A103.

13. Langowski JL, Zhang X, Wu L, et al. IL-23 promotes tumour incidence and growth. Nature. 2006;442:461-465.

14. Karin M. Nuclear factor-kappaB in cancer development and progression. Nature. 2006;441:431-436.

15. D'Haens G, Sandborn WJ, Feagan BG, et al. A review of activity indices and efficacy end points for clinical trials of medical therapy in adults with ulcerative colitis. Gastroenterology. 2007;132:763-786.

16. Truelove SC, Witts LJ. Cortisone in ulcerative colitis; preliminary report on a therapeutic trial. $\mathrm{Br}$ Med J. 1954;2:375-378.

17. Rachmilewitz D. Coated mesalazine (5-aminosalicylic acid) versus sulphasalazine in the treatment of active ulcerative colitis: a randomised trial. BMJ. 1989;298:82-86.

18. Schroeder KW, Tremaine WJ, Ilstrup DM. Coated oral 5-aminosalicylic acid therapy for mildly to moderately active ulcerative colitis. A randomized study. N Engl J Med. 1987;317:1625-1629.

19. Higgins PDR, Schwartz M, Mapili J, et al. Is endoscopy necessary for the measurement of disease activity in ulcerative colitis?. Am J Gastroenterol. 2005;100:355-361.

20. Powell-Tuck J, Bown RL, Lennard-Jones JE. A comparison of oral prednisolone given as single or multiple daily doses for active proctocolitis. Scand J Gastroenterol. 1978;13:833-837.

21. Walmsley RS, Ayres RC, Pounder RE, et al. A simple clinical colitis activity index. Gut. 1998;43:29-32.

22. Seo M, Okada M, Yao T, et al. Evaluation of disease activity in patients with moderately active ulcerative colitis: comparisons between a new activity index and Truelove and Witts' classification. Am J Gastroenterol. 1995;90:1759-1763.

23. Manes G, Imbesi V, Ardizzone S, et al. Appropriateness and diagnostic yield of colonoscopy in the management of patients with ulcerative colitis: a prospective study in an open access endoscopy service. Inflamm Bowel Dis. 2008;14:1133-1138.

24. Su C, Lewis JD, Goldberg B, et al. A meta-analysis of the placebo rates of remission and response in clinical trials of active ulcerative colitis. Gastroenterology. 2007;132:516-526.

25. Rutter M, Saunders B, Wilkinson K, et al. Severity of inflammation is a risk factor for colorectal neoplasia in ulcerative colitis. Gastroenterology. 2004;126:451-459. 